\title{
Orbiter Trajectory Analysis for a Two-Stage Reusable Launch Vehicle
}

\author{
Adam L. Cowling* \\ NASA Langley Research Center, Hampton, Virginia 23681
}

\begin{abstract}
Trajectory analysis performed on NASA's reference two-stage-to-orbit launch vehicle upper stage will be presented. The work was completed in support of the Hypersonics Multidisciplinary Analysis and Optimization effort for the NASA-Air Force Joint System Study. Three degree-of-freedom (3-DOF) untrimmed trajectory analysis was performed for the orbiter ascent, closure and re-entry. An iterative closure process resulted in a 333,000 lb initial mass for the orbiter. The re-entry trajectory satisfied heating constraints for all payload out cases and met the constraints with reduced margins for payload in cases. Abort trajectories for engine out at staging, engine out during ascent, and failure to circularize in orbit, gave insight to the robustness of the orbiter. A trimmed ascent trajectory defined an engine gimbal location and the body flap angle best suited for maximizing injected mass. A trimmed re-entry trajectory revealed a need to update the trim routine to accommodate full flap aerodynamic data.
\end{abstract}

\section{Introduction}

TRAJECTORY analysis has been performed in support of the Hypersonics Multidisciplinary Analysis and 1 Optimization (MDAO) effort for the NASA-Air Force Joint System Study (JSS). Three degree-of-freedom (3-DOF) untrimmed trajectory analysis was performed for the NASA reference two-stage-to-orbit (TSTO) vehicle. Trajectories for engine out and OMS failure scenarios were also developed. A preliminary investigation of trimmed trajectory analysis was performed to show that the vehicle is feasible for trimmed analysis. This paper will present the trajectory work completed on the TSTO upper stage orbiter for the ascent and re-entry flight phases.

\section{I.A. Background and Motivation}

The Joint System Study is a collaborative effort of NASA and the U.S. Air Force to develop and analyze reusable two-stage-to-orbit vehicle concepts in order to evaluate the critical technologies for development. The NASA horizontal launch vehicle configuration has an airbreathing turbine-based combined-cycle (TBCC) booster and an all-rocket orbiter, whereas the Air Force vertical launch configuration has an all-rocket booster and an airbreathing rocket-based combined-cycle (RBCC) orbiter. The Joint System Study vehicles serve as analytical testbeds for multiple advanced technologies, providing opportunities for tool development and validation, and supporting the development of a technology roadmap for the Air Force Chief Technologist and NASA Associate Administrator for Aeronautics Research.

\section{I.A.1. Mission Overview}

The mission objective of the JSS is to deliver a 20,000 lb payload to a $50 \mathrm{nmi} \times 100 \mathrm{nmi}$ initial injection orbit, which is circularized at apogee. The NASA reference vehicle consists of a TBCC booster stage and an all-rocket orbiter stage. The vehicle performs horizontal takeoff in a mated configuration using booster turbines and booster tail rockets. At Mach 3, the booster transitions to a dual-mode scramjet, which fires until just before stage separation. At Mach 10, 100 psf dynamic pressure, and a $5^{\circ}$ flight path angle, the two vehicles separate, with the booster returning to the launch site. The orbiter stage, shown in Fig. 1, continues a rocket ascent to a $50 \mathrm{nmi} \times 100 \mathrm{nmi}$ orbit. Orbiter re-entry is an unpowered glide back to the launch site. The trajectory work presented here details the orbiter ascent and re-entry phases of the flight.

\footnotetext{
*Aerospace Engineer, Vehicle Analysis Branch. Member AIAA.
} 


\section{I.A.2. Ground Rules and Assumptions}

The JSS guidelines provide fidelity level definitions for each discipline to follow, promoting consistency throughout the study. Level 1 fidelity for the trajectory discipline is defined as performing an optimized ascent, fly-back and re-entry 3 -DOF point mass simulation (untrimmed). Level 2 fidelity includes trim in the pitch plane.

Trajectory analysis was completed within the requirements of the ground rules and assumptions (GR\&A) set forth by the JSS leadership. The ground rules included accelerations limits of $5 \mathrm{~g}\left(160.9 \mathrm{ft} / \mathrm{s}^{2}\right)$ total, $+2.5 \mathrm{~g}\left(+80.4 \mathrm{ft} / \mathrm{s}^{2}\right)$ pullup, and $-1.5 \mathrm{~g}\left(-48.3 \mathrm{ft} / \mathrm{s}^{2}\right)$ pushover with a maximum pitch rate of $15 \%$ sec. The simulation must use a 1976

Standard Atmosphere with no winds, a WGS-84 Earth gravity model,

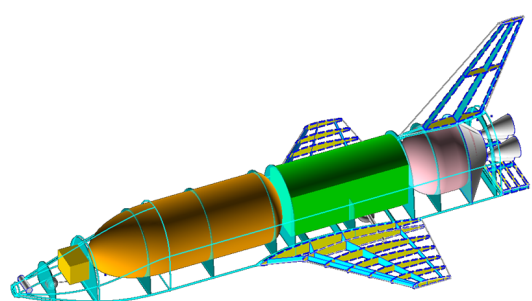

Figure 1: NASA TSTO reference orbiter. and a launch/landing at the Kennedy Space Center (KSC). Orbit insertion requirements were: injection into a $50 \mathrm{nmi} \times 100 \mathrm{nmi}$ orbit at $28.5^{\circ}$ inclination and $75 \mathrm{nmi}$ altitude. For re-entry, the orbiter must meet a maximum cross-range of $1200 \mathrm{nmi}$, and the simulation must end at $0 \mathrm{nmi}$ out and 20,000 $\mathrm{ft}$ altitude.

\section{I.A.3. Program to Optimize Simulated Trajectories II}

All trajectory simulations were generated using the Program to Optimize Simulated Trajectories II $^{1}$ (POST2). POST2 is an event driven, point mass trajectory simulation software with discrete parameter targeting and optimization (T/O) capability. POST2 provides efficient T/O capability for simulating trajectories of multiple powered or unpowered vehicles near an arbitrary rotating, oblate body using equality and inequality constraints. POST2 is equipped with generalized attracting body and vehicle models, as well as flexible input/output options. Originally developed as a Shuttle simulation code, POST2 has undergone extensive modification and validation, and has been used on multiple projects including Hyper-X, Mars Exploration Rover, and Titan IV.

\section{Level 1 Analysis}

Level 1 simulations for the orbiter included an ascent trajectory from staging to orbit, a re-entry trajectory from orbit down to 20,000 ft altitude, and abort trajectories. The abort trajectories considered failure to start an engine at staging, failure of an engine during ascent, and failure to circularize at apogee. The ascent trajectory will be presented along with sizing and closure results, followed by the re-entry and abort trajectories.

\section{II.A. Orbiter Ascent and Closure}

The orbiter ascent trajectory was the flight phase beginning from stage separation at Mach 10, 100 psf dynamic pressure, $5^{\circ}$ flight path angle and ending at a $50 \mathrm{nmi} \times 100 \mathrm{nmi}$ orbit. The orbiter was powered by four LOX/LH2 rocket engines that were on full throttle from staging to orbit insertion. Closure was achieved by iterating between trajectory and sizing simulations until the propellant fraction available (PFA) met propellant fraction required (PFR). The final closed vehicle mass was input into the POST2 model to provide the final ascent trajectory.

\section{II.A.1. POST2 Modeling}

The initial state for the orbiter ascent consisted of the staging conditions mentioned previously and the longitude and latitude of the staging event determined by a mated ascent trajectory. Vehicle aerodynamics were input in the form of lift, drag, and base drag coefficient tables as functions of Mach number, dynamic pressure, and angle of attack. The tables were produced using CBAero, ${ }^{2}$ an engineering-level software tool from NASA Ames that predicts aerodynamic environments of general vehicle configurations with estimates of base drag and aeroheating. The four LOX/LH2 engines were modeled with an Isp of $459.5 \mathrm{~s}$ for an oxidizer/fuel ratio of 5.5 and an exit area of $20.35 \mathrm{ft}^{2}$. The $\mathrm{T} / \mathrm{W}$ at staging for the orbiter was 1.25 , with rockets on full throttle for the entire flight.

The control variables for the optimization problem were the azimuth at staging, 18 pitch angle table values, and the main engine cutoff (MECO) velocity. The constraints consisted of a positive flight path 
angle at MECO to ensure insertion toward apogee, $100 \mathrm{nmi}$ mean altitude at apogee, $50 \mathrm{nmi}$ mean altitude at perigee, $75 \mathrm{nmi}$ altitude at MECO, and an orbit inclination of $28.5^{\circ}$. The optimization objective was maximum injected mass at MECO, including a 20,000 lb payload.

\section{II.A.2. Closure Process}

The orbiter ascent trajectory was used to size the vehicle based on the mass injected into orbit. An iterative process combining sizing software output and trajectory analysis was used to close the vehicle. The vehicle sizing was performed in the Exploration Architecture Model for IN-space and Earth-to-orbit ${ }^{3}$ (EXAMINE) tool. EXAMINE is an Excel-based model that uses mass estimating relationships for subsystems, vehicle geometry, packaging information, and trajectory data to scale the propellant available up to the propellant required. The closure process is shown in Fig. 2. EXAMINE calculated an initial mass and length

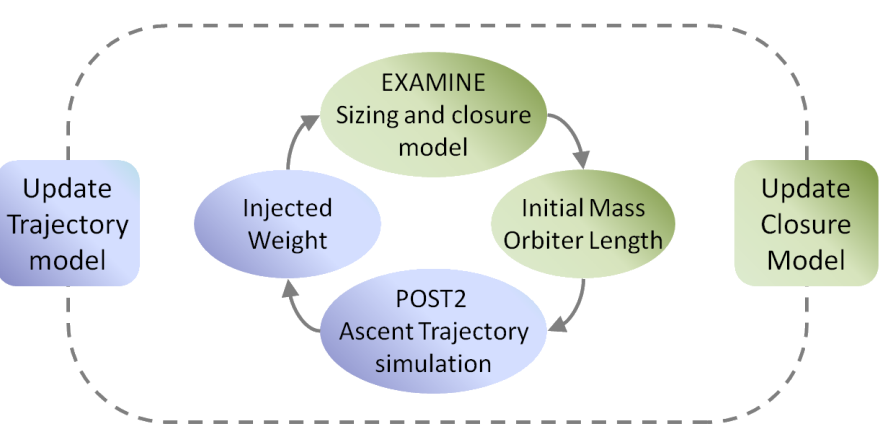

Figure 2: Iterative closure process with POST2 trajectory analysis and EXAMINE sizing and closure. estimate, given the vehicle geometry, which was input into an optimized trajectory simulation. The injected mass determined from the trajectory was input back into EXAMINE to be used in scaling the vehicle. When a closed vehicle was produced, further analysis in various disciplines, such as packaging and aerodynamics, was completed to determine if the vehicle met the requirements. If the resulting vehicle needed to be modified, the trajectory and closure models were updated to reflect the new vehicle.

The first closure iterations attempted for the orbiter are shown in Table 1. Starting from the initial size estimate, the vehicle scaled up in mass and down in length to meet the mass requirements, ultimately closing at a gross mass of $322,183 \mathrm{lb}$ and length of $104.50 \mathrm{ft}$. The closure mass was determined when the change in mass between iterations, or mass error, was less than $\pm 0.01 \%$. The first closure reached $-1 \mathrm{lb}$, or $-0.0003 \%$. Further analysis on the closed vehicle revealed that the new length did not allow enough volume

Table 1: Orbiter closure iterations.

\begin{tabular}{ccccc}
\hline Gross Mass (lb) & Injected Mass (lb) & Mass Ratio & Mass Error (lb) & Length (ft) \\
\hline 269,906 & 97,884 & 2.757 & - & 120.83 \\
310,452 & 112,431 & 2.761 & 40,546 & 103.29 \\
322,407 & 116,799 & 2.760 & 11,955 & 104.52 \\
322,184 & 116,718 & 2.760 & -223 & 104.50 \\
\hline 322,183 & 116,718 & 2.760 & -1 & 104.50 \\
\hline
\end{tabular}

for a realistic packaging layout. The unused volume provision was updated, and the vehicle length was reset to $120.83 \mathrm{ft}$ and frozen. With the outer mold line fixed, the fuel tanks were allowed to scale in length. A new set of closure iterations was performed, and the results are shown in Table 2. With the length held constant at $120.83 \mathrm{ft}$, the vehicle initial mass scaled up to $386,975 \mathrm{lb}$, closing with a mass error of $-0.0018 \%$.

Table 2: New orbiter closure iterations.

\begin{tabular}{ccccc}
\hline Gross Mass (lb) & Injected Mass (lb) & Mass Ratio & Mass Error (lb) & Length (ft) \\
\hline 322,184 & 116,718 & 2.760 & - & 120.83 \\
387,091 & 140,262 & 2.760 & 64,907 & 120.83 \\
386,982 & 140,225 & 2.760 & -109 & 120.83 \\
\hline 386,975 & 140,217 & 2.760 & -7 & 120.83 \\
\hline
\end{tabular}


As the orbiter was further analyzed, it became evident that a redesign was needed to satisfy trim performance and landing speed requirements. The initial orbiter was designated "A", and the redesigned orbiter was designated "B". The redesign of the orbiter included an area reduction and a forward-shift of the wings, as shown in Fig. 3. The orbiter mass model was updated with new geometry, new propellant tanks, new volume and mass margins, and improved mass estimating relationships.

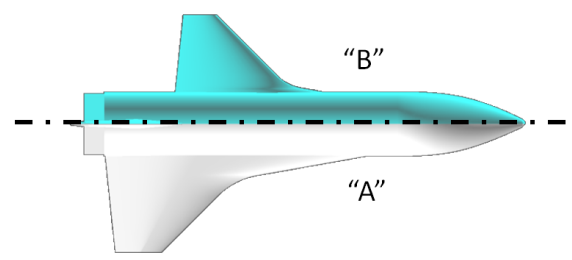

Figure 3: Original "A" orbiter and updated "B" orbiter.

\section{II.A.3. Ascent and Closure Results}

The "B" orbiter design was integrated into the POST2 and EXAMINE models, and the sizing and closure routine was performed. The final "B" orbiter closed at 333,000 lb, with an injection mass of 120,438 lb. The total flight time from staging to MECO was 234.6 seconds. The orbiter met orbit constraints and optimization tolerances with a $49.99 \mathrm{nmi} \times 100.01 \mathrm{nmi}$ orbit at $28.5^{\circ}$ inclination. Orbit insertion occurred at $74.98 \mathrm{nmi}$ altitude with a $0.374^{\circ}$ flight path angle, ensuring coast toward apogee. After orbit circularization, the on-orbit mass of the orbiter was 115,959 lb. The mass decreased to an entry mass of 113,584 lb (including payload) after a de-orbit burn. Time histories for the trajectory outputs are shown in Fig. 4. The angle of attack profile shows a smooth trend along the trajectory in Fig. 4(a). Figure 4(c) shows that the accelerations remained within the constraints set in the GR\&A.

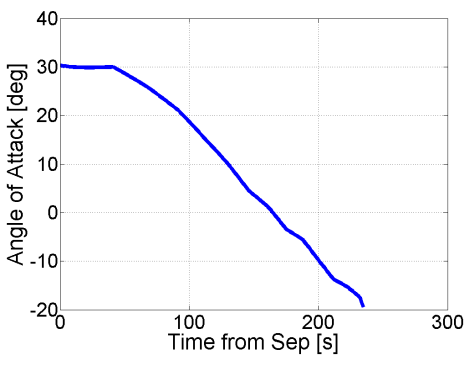

(a)

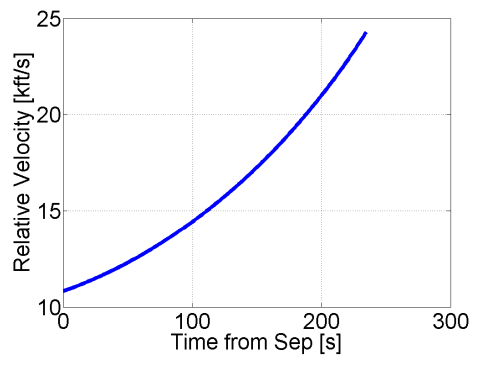

(d)

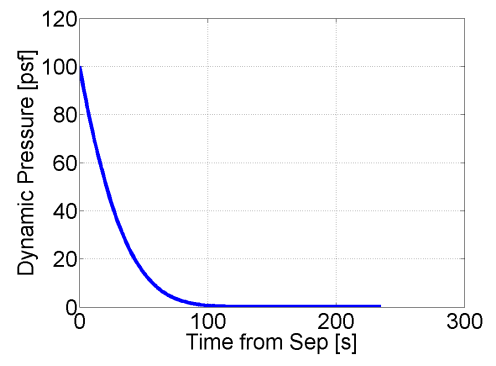

(b)

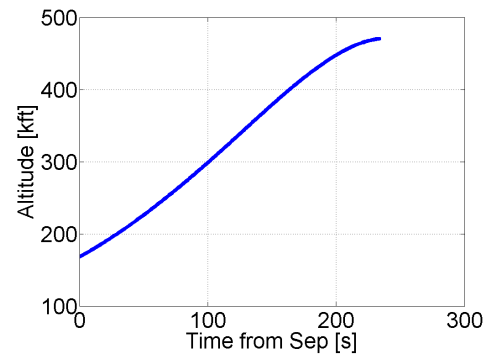

(e)

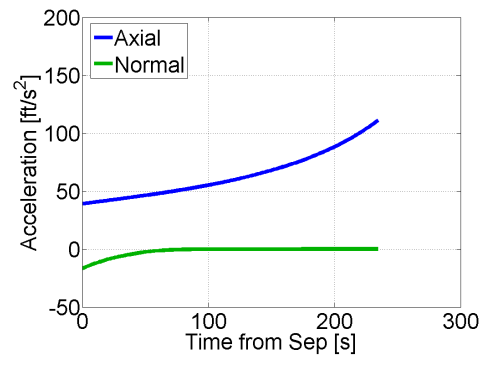

(c)

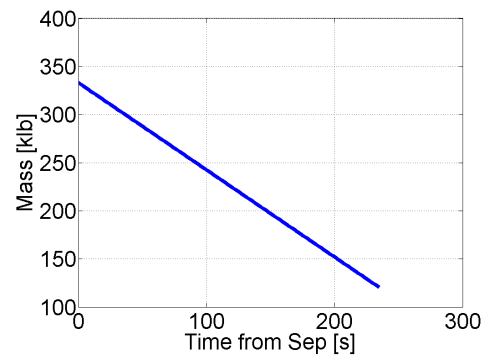

(f)

Figure 4: Orbiter ascent trajectory time histories for (a) angle of attack, (b) dynamic pressure, (c) axial and normal acceleration, (d) relative velocity, (e) altitude, and (f) mass.

\section{II.B. Orbiter Re-entry}

The orbiter re-entry trajectory began in a $100 \mathrm{nmi}$ circular orbit and consisted of a de-orbit burn, atmospheric entry at 400,000 ft and a descent to 20,000 ft. After de-orbit burn, the vehicle re-entry and descent were unpowered.

\section{II.B.1. POST2 Modeling}

The ground rules and assumptions for orbiter entry required an entry crossrange of 1200 nmi. To model the worst case scenario, the orbiter entered at 1200 nmi crossrange with the payload in. The de-orbit burn was modeled in POST2 as an instantaneous $\Delta \mathrm{V}$ addition, leaving the orbiter entry mass at $113,584 \mathrm{lb}$. In 
addition to the aerodynamic coefficient tables from the ascent deck, a CBAero-based aerothermal database was also used to model integrated aerothermal limits. This database included heat rate tables for 16 body points as functions of Mach number, dynamic pressure, and angle of attack. Several of the body points were assigned an area weight, serving as multipliers for the corresponding tables, which were summed and integrated over the trajectory. The control variables for POST2 targeting and optimization were the de-orbit burn initiation time, angle of attack, and bank angle table values as a function of velocity. The trajectory was constrained to maximum allowable heat rates based on the initial material selection (with $25 \%$ margin per the GR\&A) for the database body points with the simulation ending at zero range from KSC at 20,000 $\mathrm{ft}$ altitude. The optimization objective was to minimize the area-weighted integrated heat load.

\section{II.B.2. Re-entry Results}

The orbiter re-entry trajectory met all body point heating margins except the stagnation point (nose) and wing leading edge. The margins on those points were reduced and the trajectory was re-flown. Reducing the margins allowed for development of a best case trajectory for nose and wing leading edge heating. The updated trajectory met all constraints with a nose margin of $15 \%$ and a wing leading edge margin of $5 \%$. The orbiter re-entry ground track is shown in Fig. 5, along with a propagation of the initial orbit to illustrate the 1200 nmi crossrange constraint. The total trajectory time from entry interface (EI; 400,000 ft) to $20,000 \mathrm{ft}$ altitude was $41 \mathrm{~min}$ 29 s. Figure 6 shows the time histories for several parameters of the re-entry trajectory. The angle of attack and bank angle, shown in Fig. 6(a), were driven by the heat rate constraints. The maximum dynamic pressure experienced was $309 \mathrm{psf}$ (Fig. $6(\mathrm{~b}))$ and the maximum normal acceleration magnitude was

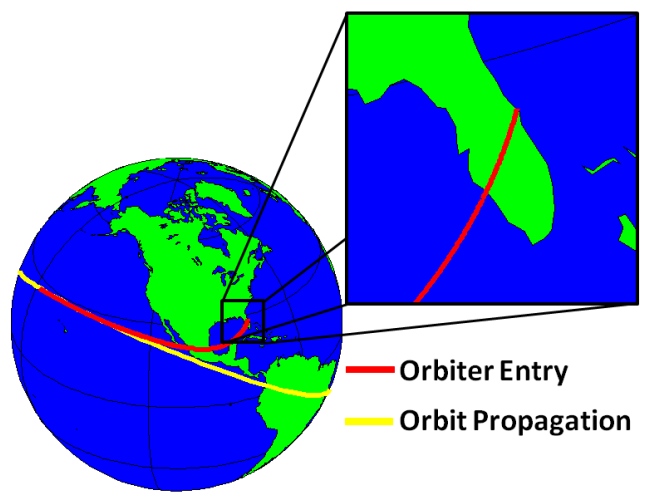

Figure 5: Ground track of orbiter re-entry trajectory showing orbit propagation and return to launch site. $44 \mathrm{ft} / \mathrm{s}^{2}$ (Fig. 6(c)), both within the GR\&A specified limits. The bumps along the altitude profile in Fig. 6(e) show where the orbiter alleviated dynamic pressure and

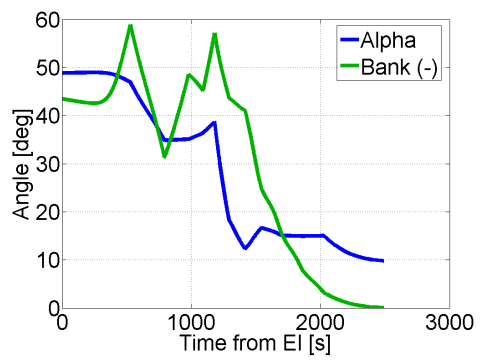

(a)

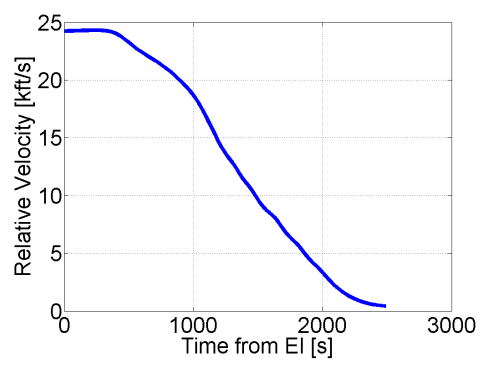

(d)

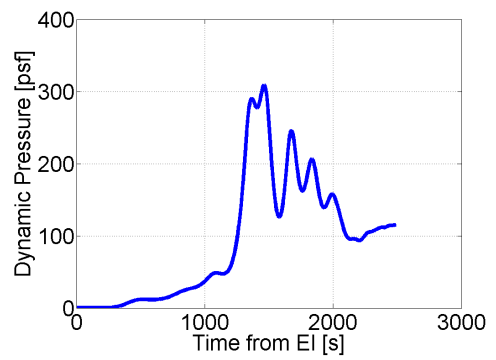

(b)

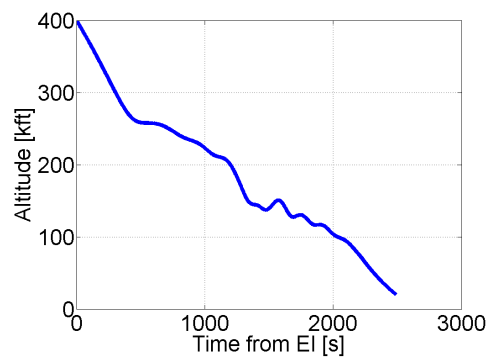

(e)

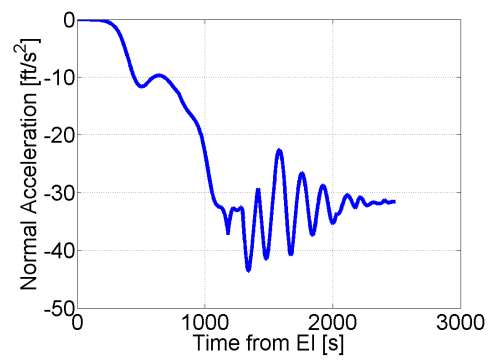

(c)

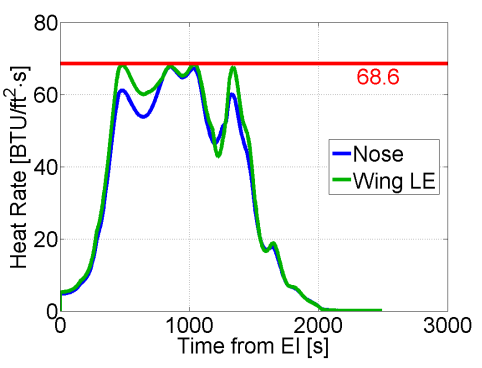

(f)

Figure 6: Orbiter re-entry trajectory time histories for (a) angle of attack and bank angle, (b) dynamic pressure, (c) normal acceleration, (d) relative velocity, (e) altitude, and (f) nose and wing leading edge heat rates with heat rate limit.

reduced the heat load by slowing the descent. Figure $6(\mathrm{f})$ shows the heat rates for the nose and wing leading 
edge, with modified margins, relative to the maximum allowable heat rate of $68.6 \mathrm{BTU} / \mathrm{ft}^{2} \cdot \mathrm{s}$.

\section{II.B.3. Entry Trade: Meeting Margins}

As a payload-in case, the baseline trajectory was also a simulation of an abort scenario. To justify a reduction in heating rate margins for the baseline case, a study was performed to look at the aeroheating levels for various entry crossranges, as well as both payload-in and payload-out options. The entry ground tracks for several entry cross range requirements are shown in Fig. 7. All of the payload-out cases satisfied

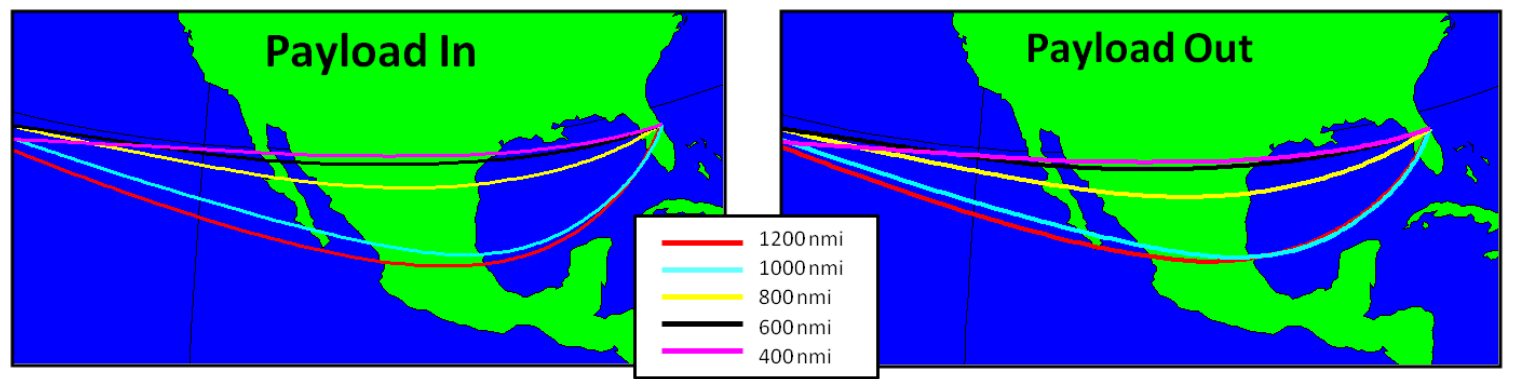

Figure 7: Ground track of orbiter re-entry trajectory for several entry crossrange values.

heating rate constraints with full margins, while only the $600 \mathrm{nmi}$ and $400 \mathrm{nmi}$ payload-in cases performed as well. The two body point constraints with the highest heating rates, the nose and the wing leading edge (LE), were unable to be met with full margins on the $1200 \mathrm{nmi}, 1000 \mathrm{nmi}$, and $800 \mathrm{nmi}$ cases. The best heating margins for these points in the payload-in trajectories are shown in Fig. 8(a). The minimum integrated heat loads for all entry cases are shown in Fig. 8(b). The development of these cases provided a distinction between abort/down-mass (payload-in) and delivery (payload-out) scenarios. This distinction implied that abort scenarios were possible with the current orbiter but were more likely to push on a heating limit.

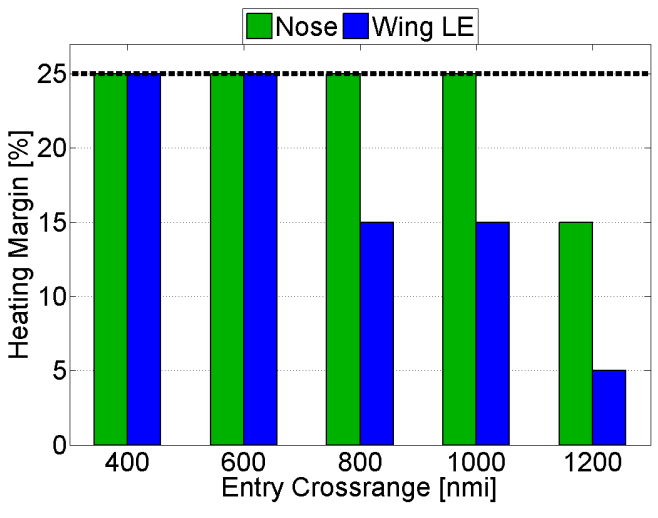

(a)

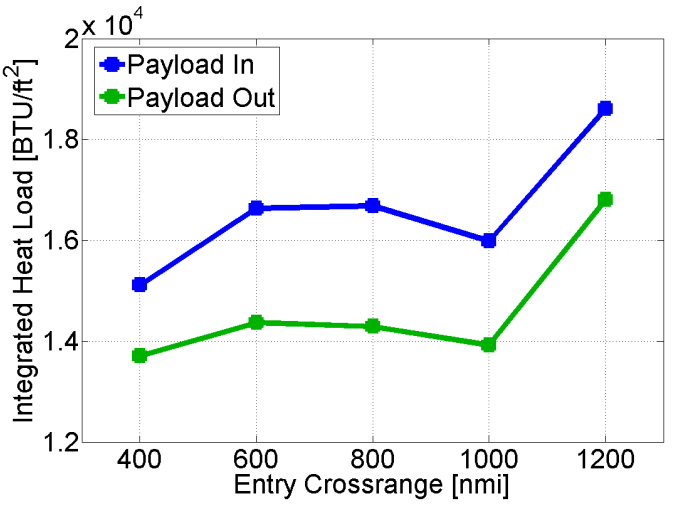

(b)

Figure 8: (a) Maximum nose and wing leading edge heating rate margins and (b) integrated heat load obtained at several entry crossrange values.

\section{II.C. Orbiter Abort Scenarios}

In support of a robust, reliable orbiter design, several abort trajectories were developed. The orbiter abort scenarios addressed were: one engine out at staging, one engine out during ascent, and a failure to circularize.

\section{II.C.1. Engine Out at Staging}

In the first abort scenario, one of the four orbiter engines failed just after separation. The goal of analyzing this scenario was to determine if the orbiter could reach a high enough orbit to be able to use the OMS to boost to a sustainable orbit. The assumptions for this trajectory were that only one engine failed with the 
remaining three maintaining nominal performance and that failure occurred after a clean separation from the booster. It is also assumed that the nominal mission could not be met. In addition, no resultant pitch or yaw effects from asymmetric thrust were modeled. Two separate trajectories were developed based on the nominal $50 \mathrm{nmi} \times 100 \mathrm{nmi}$ target orbit. The first abort case holds mean perigee altitude at $50 \mathrm{nmi}$, while maximizing the mean apogee altitude. The second abort case holds mean apogee altitude at $100 \mathrm{nmi}$, while maximizing the mean perigee altitude. Both cases were constrained to maintain an inclination of $28.5^{\circ}$, to inject the same mass to orbit as the nominal ascent, and to enter the orbit at an altitude of at least 400,000 $\mathrm{ft}$ to exit the atmosphere. The two cases produced final orbits of $50 \mathrm{nmi} \times 75 \mathrm{nmi}$ and $35 \mathrm{nmi} \times 100 \mathrm{nmi}$. Figure 9(a) shows the altitude time histories for the nominal, $50 \mathrm{nmi} \times 75 \mathrm{nmi}$, and $35 \mathrm{nmi} \times 100 \mathrm{nmi}$ orbits. The abort trajectories followed a lower path and reached orbit at 400,000 ft. Once out of the atmosphere, it was assumed that the orbit could be sustained to apogee and that the orbiter had sufficient OMS fuel to maneuver onto a path for re-entry. The mass profiles are shown in Fig. 9(b). All trajectories injected the same mass, according to the constraints, though the abort cases took longer because of lower thrust.

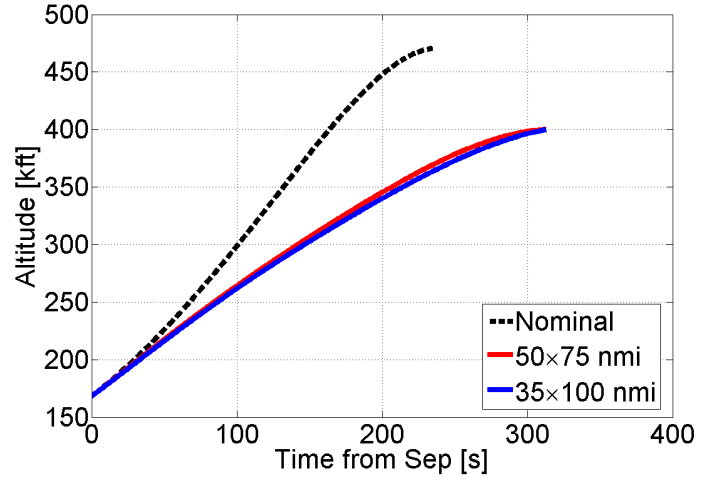

(a)

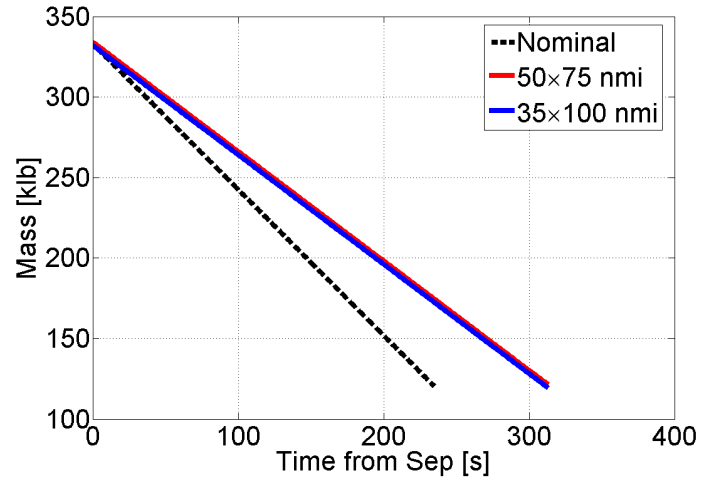

(b)

Figure 9: Engine out at staging abort trajectory time histories for (a) altitude and (b) mass.

\section{II.C.2. Engine Out on Ascent}

The second abort case assumed successful separation and ignition of all four orbiter engines with a single engine out event occurring during ascent. With the orbiter's capability to reach an orbit from staging with engine loss as described above, it became important to determine the point on ascent where the orbiter no longer needs to abort to orbit. This case was designed as an off-nominal trajectory where the orbiter could lose one engine and still meet mission parameters. The trajectory was constrained to reach a $50 \mathrm{nmi} \times 100$ $\mathrm{nmi}$ orbit at a positive flight path angle, an altitude of $75 \mathrm{nmi}$, and an inclination of $28.5^{\circ}$. By maximizing mass to orbit and iterating with engine cutoff time, the minimum time into ascent that the orbiter could lose an engine and make the nominal mission was determined to be $47 \mathrm{~s}$. Figure 10(a) shows the altitude profile

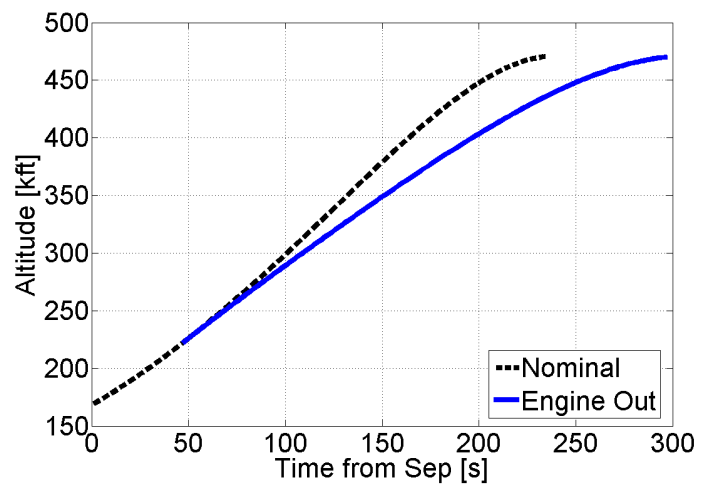

(a)

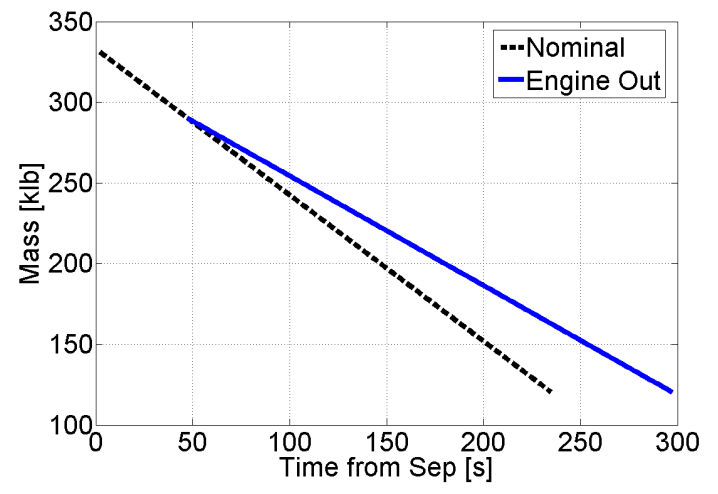

(b)

Figure 10: Engine out on ascent abort trajectory time histories for (a) altitude and (b) mass. 
for the nominal and engine out cases. The engine out case took about $60 \mathrm{~s}$ longer to reach the nominal orbit. Figure 10(b) shows the mass profiles, illustrating the mass difference starting at $47 \mathrm{~s}$.

\section{II.C.3. Failure to Circularize}

The third orbiter abort scenario simulated was the failure of the OMS at circularization into a $100 \mathrm{nmi}$ orbit. In this case, it was important to show that the orbiter could continue on the $50 \mathrm{nmi} \times 100 \mathrm{nmi}$ orbit, completing a once-around. Upon reaching entry interface after apogee, the orbiter returned to KSC as in the nominal re-entry trajectory. Figure 11 shows the ground track of the once-around and re-entry. The orbiter ascended from staging, completed main engine cutoff (MECO), and reached apogee as in the nominal mission. At apogee, the orbiter failed to circularize and continued on the elliptical orbit, descending toward the atmosphere. At 400,000 ft, the orbiter re-entered the atmosphere, using bank angle and angle of attack commands to stay under thermal constraints. The orbiter successfully completed a once-around re-entry, meeting the same thermal constraints as the nominal re-entry trajectory.

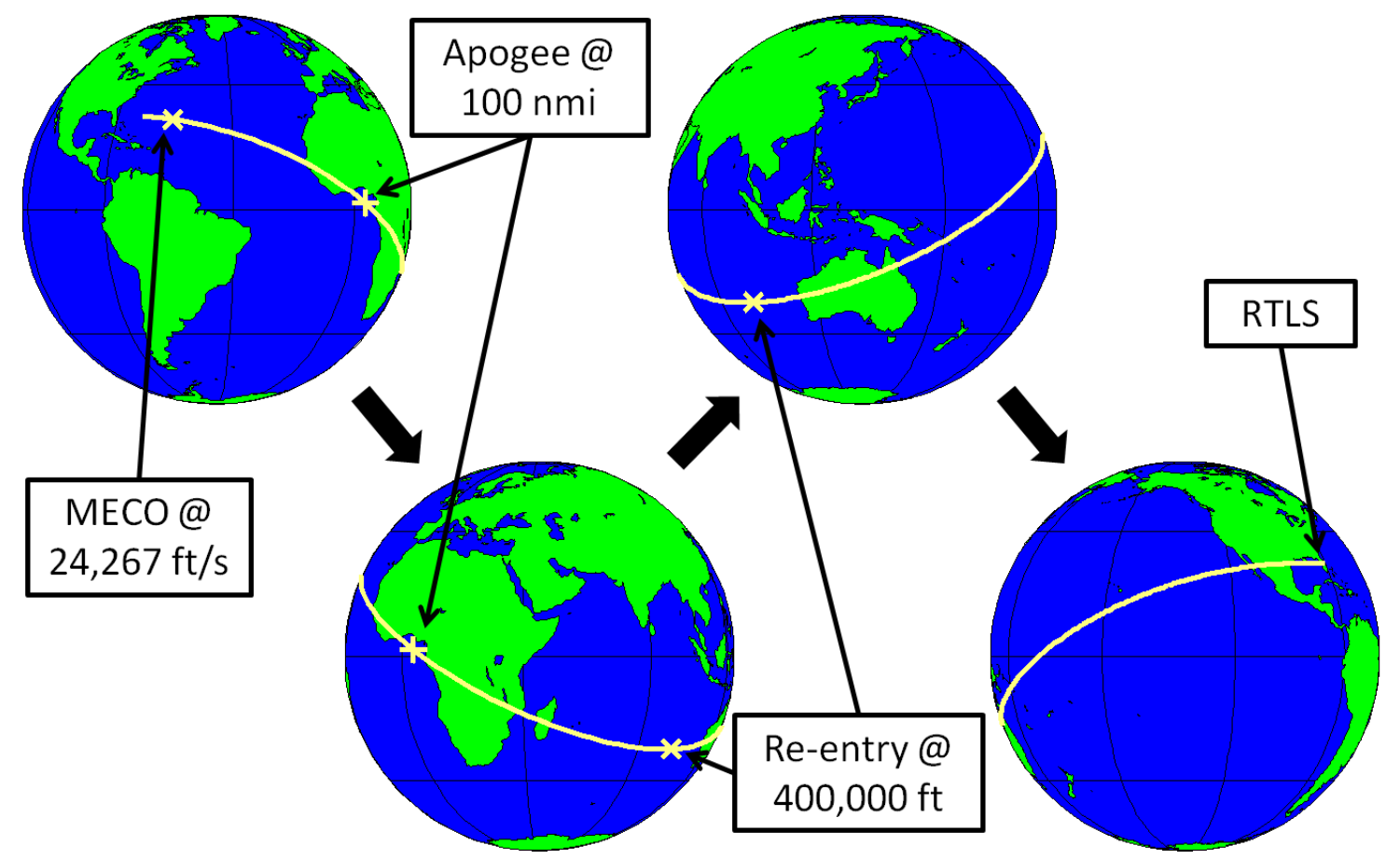

Figure 11: Ground track of orbiter ascent and re-entry trajectory for abort due to failure to circularize orbit.

\section{Level 2 Analysis}

For the Joint System Study, trajectory analysis is required to include static trim calculations in the pitch plane for Level 2. Preliminary Level 2 analysis has been performed on the orbiter ascent and re-entry trajectories. For the orbiter ascent, a trade study was developed to look at the pitch trim effects of thrust gimbaling and body flap deflections on injected mass. A pitch trim plan for the orbiter re-entry was developed to integrate RCS thrusters for high-altitude trim, a body flap schedule for gross trim, and elevons for fine trim.

\section{III.A. Trimmed Ascent}

This preliminary trimmed ascent analysis served as a feasibility study to show the Level 1 vehicle was suitable for Level 2 analysis. A trade study was performed on thrust incidence angle and injected mass resulting from body flap deflections and gimbal point offsets. The set of vertical gimbal offset values for 
the study was in $0.25 \mathrm{ft}$ increments from $-3 \mathrm{ft}$ to $+0.25 \mathrm{ft}$ with respect to the CG. The range of body flap deflections varied every $2.5^{\circ}$ from $-10^{\circ}$ to $+30^{\circ}$. A trajectory optimization was performed for all 238 combinations. A surface plot, shown in Fig. 12, represents the resulting injection masses and total thrust incidence ranges for all cases. In order to move along with the orbiter design, the baseline case for future trimmed analysis was chosen as that with the largest injected mass, given design constraints. A hard limit of $\pm 2^{\circ}$ deflection range was the first requirement. Additionally, the structural/geometric feasibility of the engine gimbal point required an offset of no more than $\pm 1 \mathrm{ft}$. The chosen case had a gimbal point vertically located 9 inches below the initial orbiter CG and a body flap deflection of $10^{\circ}$ trailing edge down. The offset put the gimbal point in a vertical location enclosed by the initial and final CG locations, allowing for a smaller range of incidence angles. The final incidence range was $\pm 1.99^{\circ}$, and the final injected mass was $97,884 \mathrm{lb}$.

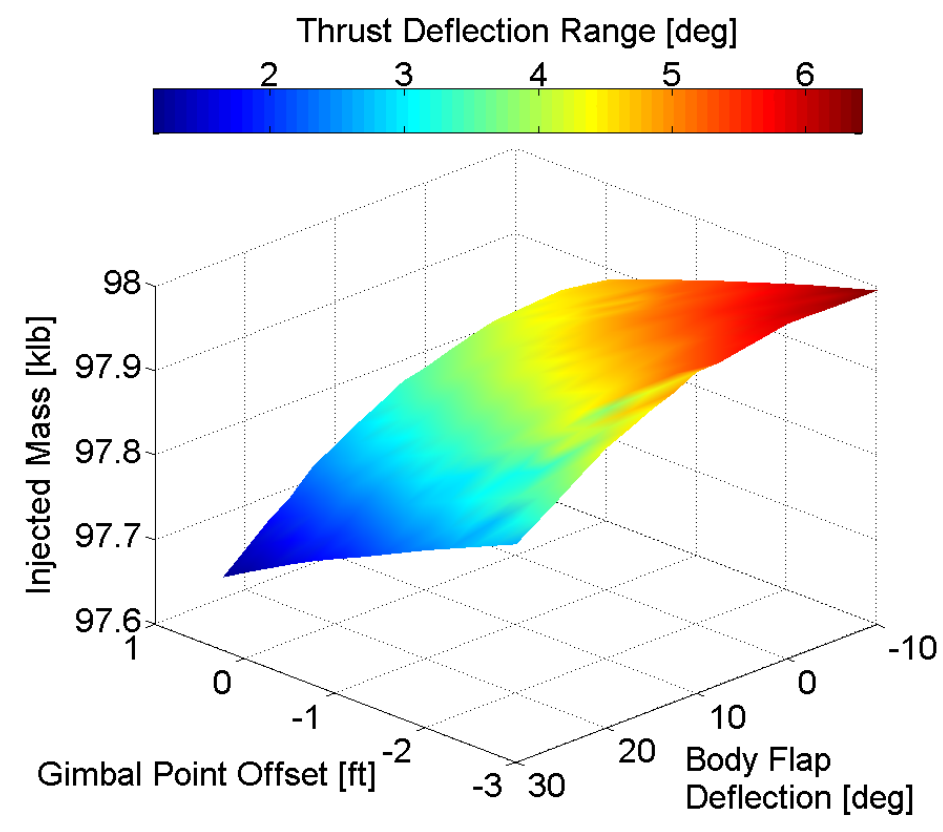

Figure 12: Trade study surface showing thrust deflection range and injection mass for varied body flap deflection and vertical gimbal offset.

\section{III.B. Trimmed Re-entry}

For the orbiter re-entry, a trim plan was developed to balance the moments in the full set of flight regimes encountered by the vehicle. From EI to a dynamic pressure of 20 psf, a RCS system based on the Space Shuttle was implemented. For the remaining glideback, a combination of body flap gross trim and elevon fine trim was tested.

\section{III.B.1. RCS Trim}

For high altitude trim from entry interface down to 20 psf dynamic pressure, a reaction control system (RCS) was integrated into the orbiter re-entry trajectory deck. The RCS was based heavily on the Shuttle RCS thrusters. For pitch trim, six aft thrusters pointing upward ware used to balance the nose-down moment. Each thruster had a specific impulse of $289 \mathrm{~s}$, a vacuum thrust of $870 \mathrm{lb}$, and a nozzle exit area of $2.27 \mathrm{ft}^{2}$. The major assumptions with this simulation were that the thrusters could be throttled (as opposed to a bang-bang control) and that the thrusters could be on for the entire flight from entry interface to 20 psf. The assumptions were made to suit 3-DOF analysis, as a RCS is typically a 6-DOF application. The total moment of the untrimmed trajectory is shown in Fig. 13(a), along with the RCS enabled trajectory with the moment trimmed out. The throttle value of the thrusters is shown in Fig. 13(b), reaching a maximum of $57 \%$, with a total propellant usage of $2,397 \mathrm{lb}$. 


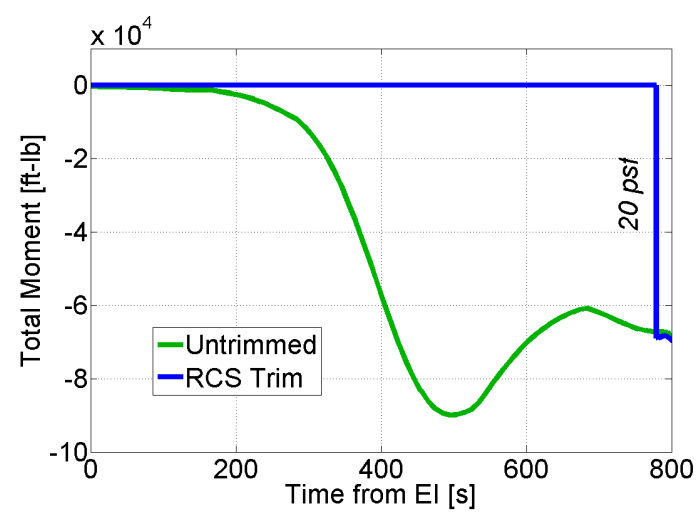

(a)

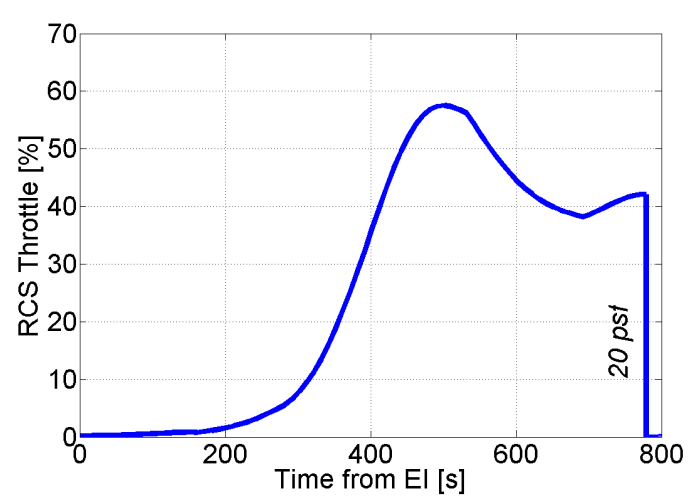

(b)

Figure 13: (a) Total moment for untrimmed and RCS-trimmed entry trajectories and (b) RCS throttle level from entry interface to 20 psf dynamic pressure.

\section{III.B.2. Body Flap Gross Trim}

At 20 psf, the RCS was turned off and the orbiter control surfaces became active. The orbiter body flap was used for gross trim and the elevons were used for the remaining moment. In order to perform gross trim with the body flap, an optimization strategy was employed to reduce the moment. The optimization objective was to minimize the integral of the moment. The variables in the optimization were values from a body flap table schedule. Figure 14(a) shows the reduced moment compared to the total moment for the untrimmed nominal case. The body flap deflection history is shown in Fig. 14(b). The body flap was constrained to $\pm 20^{\circ}$.

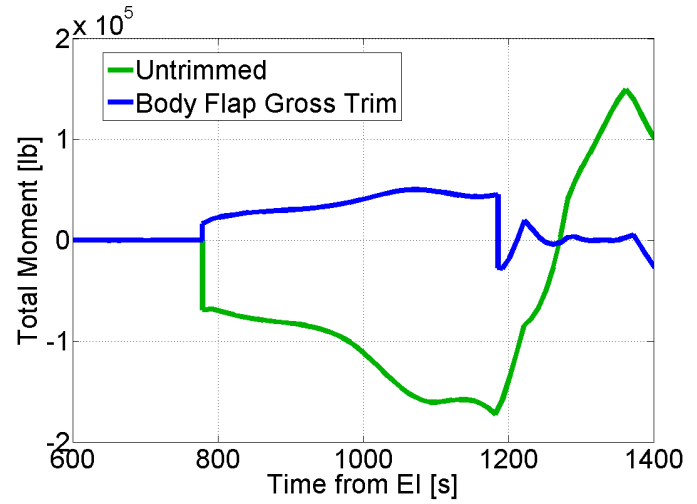

(a)

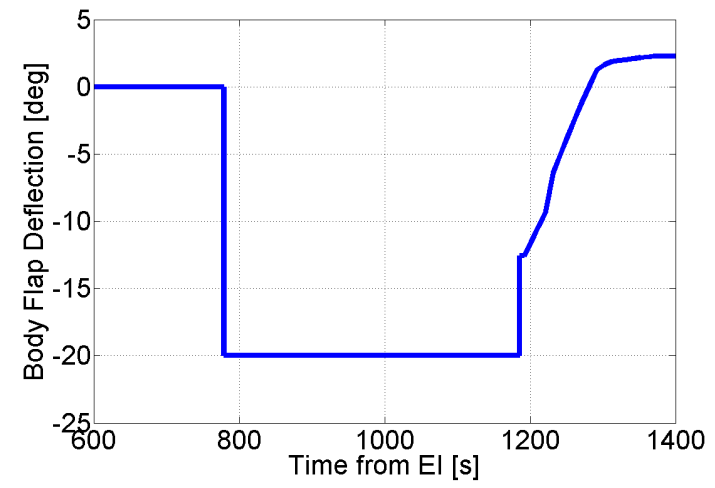

(b)

Figure 14: (a) Total moment for untrimmed and body flap-trimmed entry trajectories and (b) body flap deflection.

\section{III.B.3. Flap Fine Trim}

The trim plan called for elevons to trim out the remaining moment, but the standard POST2 flap trim routine had not been updated to allow for the level of flap aerodynamic data that was produced. The standard POST2 trim routine accepted inputs in the form of lift coefficient increments per degree. The problem with this method was that the flap deflection angle could not be used as a table lookup input, forcing the user to create a constant increment-per-degree table. Figure 15 illustrates the need for an updated trim routine. The actual data provided - this example at Mach 6, $200 \mathrm{psf}$, and $15^{\circ}$ - was nonlinear. In order to create a constant increment-per-degree, an average increment-per-degree was created to serve in place of the actual data. For comparison, the minimum and maximum increment-per-degree values (for each Mach number-dynamic pressure-angle of attack set) were also plotted. The resulting plot shows that the 
minimum increment-per-degree was the best solution for elevon deflections below $-10^{\circ}$; the average was best for the $-10^{\circ}$ to $0^{\circ}$ range; and the maximum was best for positive deflections up to $25^{\circ}$. Above $25^{\circ}$, it was not possible to accurately simulate the trend of the data. To produce a true trimmed trajectory using the best data available, a trim routine update needs to be integrated into POST2 to allow variable increment tables across the full range of flap deflections.

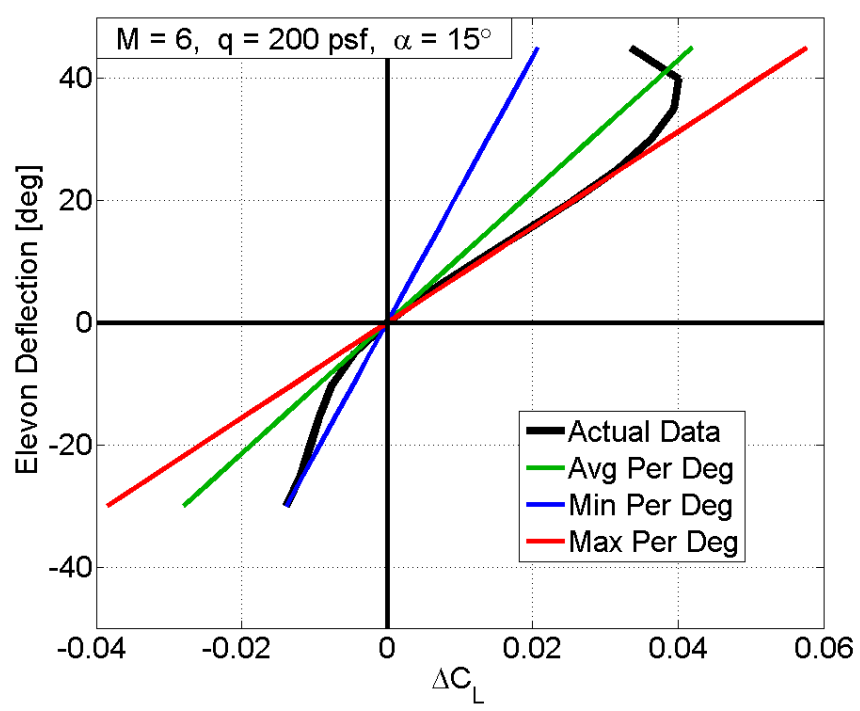

Figure 15: Example of linearization of elevon aerodynamic coefficient effects for Mach 6,200 psf dynamic pressure, and $15^{\circ}$ angle of attack.

\section{Conclusion}

Trajectory analysis has been performed in support of the Hypersonics Multidisciplinary Analysis and Optimization effort for the NASA-Air Force Joint System Study. Ascent, re-entry, and abort trajectories have been developed for a two-stage-to-orbit launch vehicle upper stage orbiter. An iterative closure process resulted in a 333,000 lb initial mass for the orbiter. The re-entry trajectory satisfied heating constraints for all payload out cases and met the constraints with reduced margins for payload in cases. Abort trajectories for engine out at staging, engine out during ascent, and failure to circularize in orbit, gave insight to the robustness of the orbiter. A preliminary investigation of Level 2 trimmed trajectory analysis was performed to show that the Level 1 vehicle was suitable for Level 2 analysis. The trimmed ascent trajectory defined an engine gimbal location and the body flap angle best suited for maximizing injected mass. The trimmed re-entry revealed a need to update the POST2 trim routine to accommodate full flap aerodynamic data.

\section{Acknowledgments}

The author thanks D.R. Komar for his sizing and closure analysis in EXAMINE, Jeff Bowles for providing all CBAero aerodynamic and aerothermal databases, and Jeff Robinson for mentorship and leadership in the MDAO effort.

\section{References}

\footnotetext{
${ }^{1}$ Striepe, S.A., et al., "Program to Optimize Simulated Trajectories (POST II) Volume II: Utilization Manual," Version 1.1.6.G, 2004.

${ }^{2}$ Kinney, D.J., "Aero-Thermodynamics for Conceptual Design", 42nd AIAA Aerospace Sciences Meeting and Exhibit, Reno, NV, 2004.

${ }^{3}$ Komar, D.R., Hoffman, J., Olds, A., and Seal, M., "Framework for the Parametric System Modeling of Space Exploration Architectures," AIAA-2008-7845, AIAA SPACE 2008 Conference and Exposition, San Diego, CA, 2008.
} 